\title{
Web-Based Technology for Remote Viewing of Radiological Images: App Validation
}

${\text { Qiusha } \text { Min }^{1 *}, \mathrm{PhD} \text {; Xin Wang }}^{1^{*}}, \mathrm{BSc}$; Bo Huang ${ }^{2 *}, \mathrm{MBBS}$; Liangzhou $\mathrm{Xu}^{2}, \mathrm{MBBS}$

${ }^{1}$ School of Educational Information Technology, Central China Normal University, Wuhan, Hubei, China

${ }^{2}$ Department of Radiology, Wuhan Hospital of Traditional Chinese Medicine, Wuhan, China

*these authors contributed equally

\section{Corresponding Author:}

Qiusha Min, PhD

School of Educational Information Technology

Central China Normal University

No.152 Luoyu Road

Wuhan, Hubei, 430079

China

Phone: 862767867597

Email: qiusham@mail.ccnu.edu.cn

\section{Abstract}

Background: Internet technologies can create advanced and rich web-based apps that allow radiologists to easily access teleradiology systems and remotely view medical images. However, each technology has its own drawbacks. It is difficult to balance the advantages and disadvantages of these internet technologies and identify an optimal solution for the development of medical imaging apps.

Objective: This study aimed to compare different internet platform technologies for remotely viewing radiological images and analyze their advantages and disadvantages.

Methods: Oracle Java, Adobe Flash, and HTML5 were each used to develop a comprehensive web-based medical imaging app that connected to a medical image server and provided several required functions for radiological interpretation (eg, navigation, magnification, windowing, and fly-through). Java-, Flash-, and HTML5-based medical imaging apps were tested on different operating systems over a local area network and a wide area network. Three computed tomography colonography data sets and 2 ordinary personal computers were used in the experiment.

Results: The experimental results demonstrated that Java-, Flash-, and HTML5-based apps had the ability to provide real-time 2D functions. However, for 3D, performances differed between the 3 apps. The Java-based app had the highest frame rate of volume rendering. However, it required the longest time for surface rendering and failed to run surface rendering in macOS. The HTML5-based app had the fastest surface rendering and the highest speed for fly-through without platform dependence. Volume rendering, surface rendering, and fly-through performances of the Flash-based app were significantly worse than those of the other 2 apps.

Conclusions: Oracle Java, Adobe Flash, and HTML5 have individual strengths in the development of remote access medical imaging apps. However, HTML5 is a promising technology for remote viewing of radiological images and can provide excellent performance without requiring any plug-ins.

(J Med Internet Res 2020;22(9):e16224) doi: 10.2196/16224

\section{KEYWORDS}

internet access; medical informatics applications; computer-assisted image analyses; computer-assisted three-dimensional imaging; medical imaging; radiology; application

\section{Introduction}

Recently, modern technology has made it possible to generate digital images using medical equipment. Compared with traditional film-based images, these types of images have several advantages (eg, they are easy to share, transmit, and process) [1]. These advantages promote the popularity of the digital imaging systems in hospitals all over the world, and offer the 
possibility for remote viewing and processing. However, the successful implementation of a teleradiology system requires a fast network and easy access [2]. If the system does not meet these requirements, radiologists may be reluctant to use the teleradiology system.

Internet technologies can create advanced and rich web-based apps that allow radiologists to easily access teleradiology systems and remotely view medical images. Compared with picture archiving and communication systems or other imaging workstations which require dedicated hardware and software, a web-based app is easy to set up and has a low cost [3]. These apps can be run on almost all personal computers without the need for powerful equipment on the client side. There are 3 major internet technologies, Oracle Java [4], Adobe Flash [5], and HTML5 [6], to create these apps. In the past few decades, these 3 technologies have been used in the field of medical imaging [7-16]; however, each technology has its own drawbacks. For example, plug-ins are required by Java and Flash. Regarding HTML5, the level of support and expected performance vary depending on the browser. Thus, it is difficult to balance the advantages and disadvantages of these internet technologies and identify an optimal solution for the development of medical imaging apps. Owing to the significant growth of teleradiology and web-based radiology subspecialty training, there is a need for quantitative and qualitative evaluations of different internet technologies in the field of medical imaging $[17,18]$.

In this study, we used different technologies-Java (version 8; Oracle Corporation), Adobe Flash (version 32; Adobe Inc), and HTML5 (version 5.3; World Wide Web Consortium)- to develop web-based medical imaging apps. Subsequently, experiments were conducted to demonstrate the performance of these apps. Accordingly, the primary aim of this study was the evaluation of the performance of medical imaging apps developed with Oracle Java, Adobe Flash, and HTML5 in various scenarios. We also aimed to analyze the advantages and disadvantages of these technologies in the field of medical imaging. We believe these performance comparisons can guide developers in their efforts to identify suitable technologies to create web-based medical imaging apps, thus allowing radiologists to visualize and interpret images remotely, quickly, and effortlessly.

\section{Methods}

\section{App Design}

Medical imaging apps have several basic functions. First, the app needs to interact directly with the local file system to avoid network latency. The user can then use various 2D image processing tools, such as zooming and windowing, to identify useful information contained in the 2D image. In addition, the interpretation may be supported by 3D functions so that the volumetric data set can provide additional information on the anatomy or pathology of the patient [19]. Consequently, comprehensive medical imaging apps should meet the following requirements: (1) interact with local file systems, (2) have basic $2 \mathrm{D}$ image processing functions, and (3) allow 3D visualization of selected regions of interest in the data sets.
In this study, 3 demo apps for computed tomography (CT) colonography (also known as virtual colonoscopy) were developed using Oracle Java, Adobe Flash, and HTML5. These apps were designed to satisfy the aforementioned criteria, and they were used to evaluate Oracle Java, Adobe Flash, and HTML5 as tools to determine the best architecture for the development of a medical imaging app.

These apps provide remote access in such a way that radiologists can view images from a downloaded data set and manipulate them using 2D or 3D functions. They can be placed as a client component in a large teleradiology system. This study focuses solely on the client app and presents a comparison of 2D and 3D performance of the Oracle Java, Adobe Flash, and HTML5 technologies for the development of such medical imaging apps.

\section{Operational Flow of Radiological Interpretation Using Demo Apps}

\section{Step 1}

Select a data set to be interpreted and click the Download button to activate the download process. The selected data set is now stored on the local computer.

\section{Step 2}

The first slice of the data set is automatically displayed on the screen in the Java-based app. In the cases of the Flash and HTML5 apps, users need to click the Choose file button and select in the dialog box the downloaded file in either of the 2 apps. Thereafter, the first slice of the data set is displayed.

\section{Step 3}

Navigate through the image data set using the Previous and Next buttons or mouse scrolling.

\section{Step 4}

Interpret the data set using 2D image processing tools, such as zoom in, zoom out, and windowing.

\section{Step 5}

Interpret the data set using 3D visualization tools, such as $3 \mathrm{D}$ rendering and fly-through.

\section{App Implementation}

\section{Access to the Local File System}

All 3 apps enabled the user to choose a CT colonography data set for study (Figure 1). The selected data set was then transmitted to the client and stored on the local computer using a custom data format. Currently, Oracle Java, Adobe Flash, and HTML5 use different local file reading and writing technologies. Oracle Java downloaded the file via HTTP using the Java HttpURLConnection class. This class was used to read and write the resources referenced by a URL (uniform resource locator). Once the download was completed, the RandomAccessFile class was used to read local files in the Java-based app. Adobe Flash used the FileReference class to provide a safe way to directly read and write data to the local system (provided that the action was sanctioned by the user). Using this class in the app, the study data set was stored on the local computer disk and could then be navigated easily and 
efficiently. HTML5 had a new input type <input type = "file"> which provided a standard way to interact with local files. After reading the downloaded file, the first slice in the data set was automatically displayed on the screen.

Figure 1. Graphical user interface used to display the first slice in the data set: (left) Java, (center) Flash, and (right) HTML5.

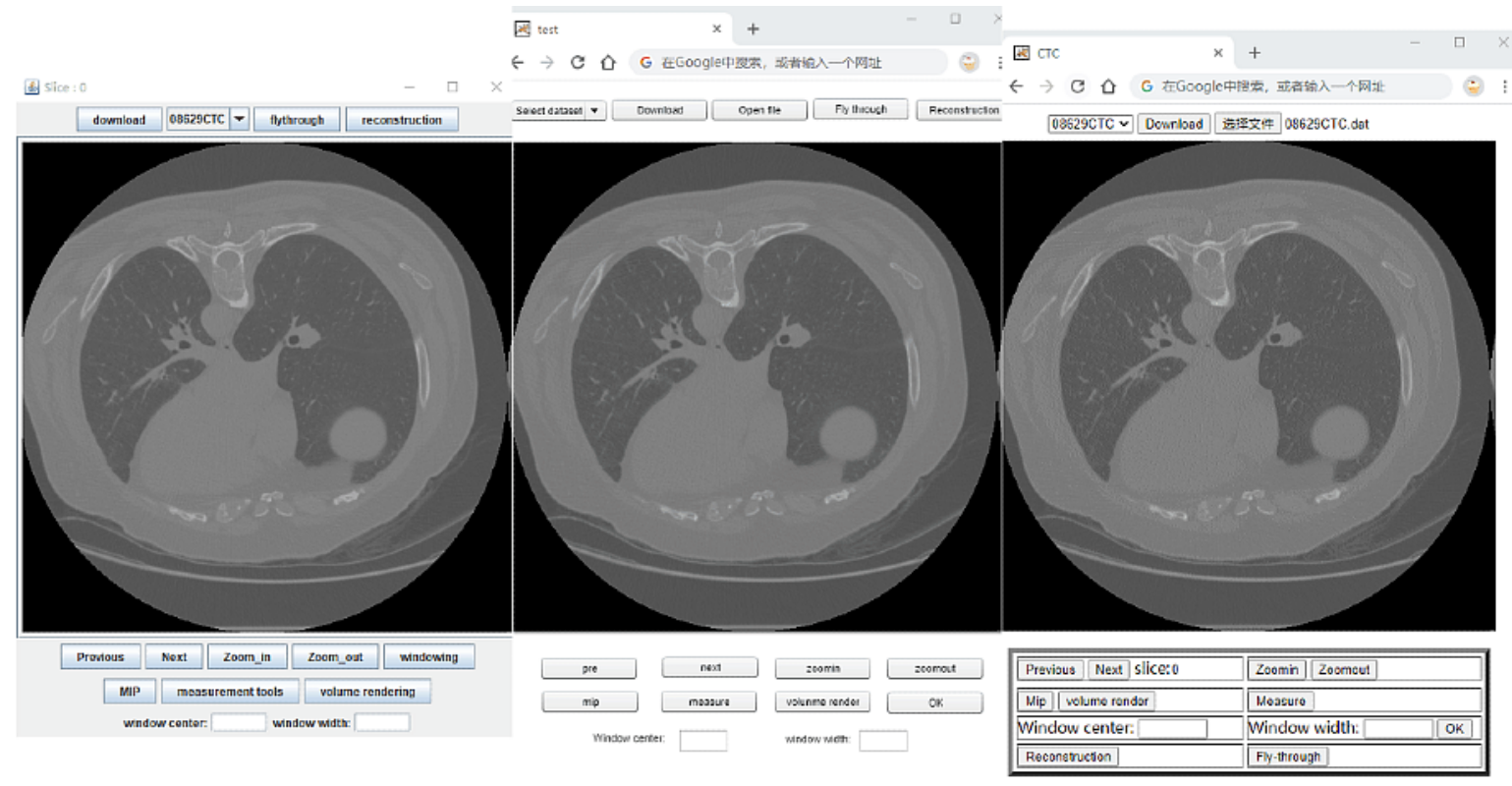

\section{Image Processing}

Another crucial requirement for these apps was pixel-level operation. For medical imaging apps, basic 2D image processing functions include magnification and windowing. For the magnification function, Java used scaleX() and scale $Y()$ in the ScalePane class to zoom in and zoom out. Flash used the Zoom class to zoom in or out of the object. HTML5 used the canvas drawImage() method to zoom in and out. In terms of windowing, Java used setRGB() to set the pixels in BufferedImage to the specified RGB value. Flash was implemented using the BitmapData class. The setPixel() and getPixel() methods in the
BitmapData class were used to change the value of each pixel in the image. For HTML5, a <canvas> element that has the ability to define the color of the pixels was used.

Figure 2 depicts a series of screenshots of the user interfaces of the windowing function in the 3 tested apps. The image is a windowed slice with the following parameters: the center of the window is $20 \mathrm{HU}$ (where HU is Hounsfield units) and the width of the window is $200 \mathrm{HU}$. Figure 3 is a screenshot of the user interface of maximum intensity projection in Java-, Flashand HTML5-based apps. Measurement and magnification functions can also be implemented by these 3 apps.

Figure 2. An anatomical axial slice at the thoracic level, wherein the center parameter is $20 \mathrm{HU}$, and the width is $200 \mathrm{HU}$. (left) Java, (center) Flash, and (right) HTML5. Screen resolution is $1920 \times 1080$.

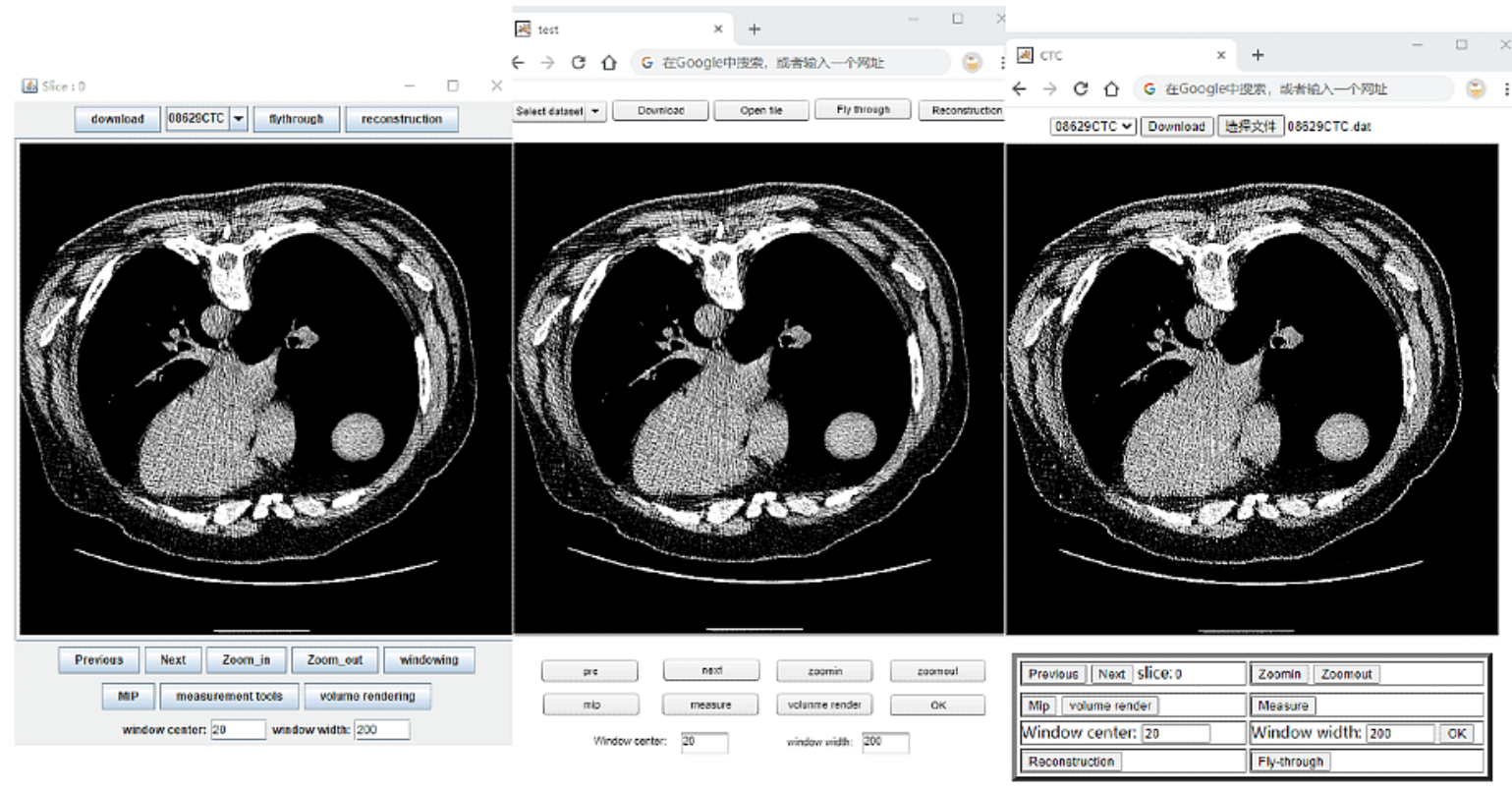


Figure 3. Graphical user interface and demonstration of maximum intensity projection. (left) Java, (center) Flash, and (right) HTML5.
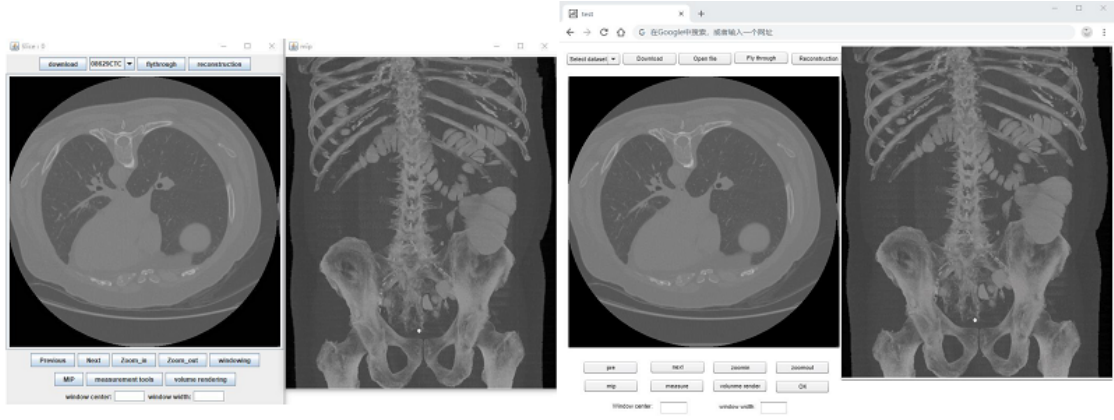

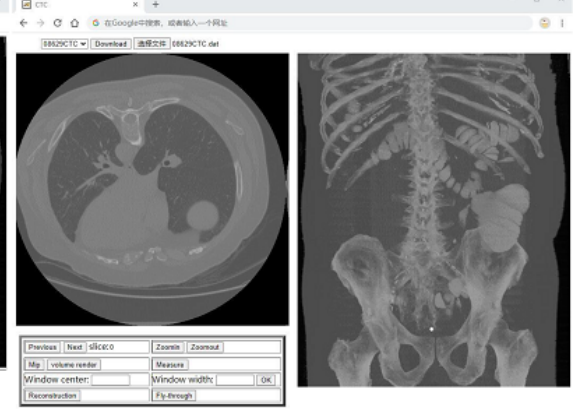

\section{$3 D$ Visualization}

It is well known that $3 \mathrm{D}$ visualization is a computationally intensive task. Hence, this work is typically implemented at a workstation equipped with a high-performance graphics processing unit (GPU). However, based on our previous research $[11,13,15]$, it is feasible to implement $3 \mathrm{D}$ visualization on a personal computer using Oracle Java, Adobe Flash, or HTML5. In this implementation, 3D visualization was based on both surface rendering and volume rendering.

Surface rendering generally involved 2 stages: surface extraction and $3 \mathrm{D}$ rendering. The marching cubes algorithm was used to extract the isosurface from a volumetric data set [20]. The information of the extracted surface (ie, the vertex and the normal) were stored on a server. Once the user sent the request to view the 3D data, the corresponding vertex and normal files were sent to the client. Subsequently, the client side was responsible for rendering the 3D model surface. Currently, Oracle Java, Adobe Flash, and HTML5 enable the provision of hardware $3 \mathrm{D}$ rendering, which is a fast rendering mode when compared to that of software rendering. Java used Canvas3D to implement 3D rendering. For Adobe Flash, a Context3D object was used in its 3D app programming interface (API). Using the createVertexBuffer() method, Flash could send the vertices and normals to the GPU directly and perform a fast reconstruction. The combination of HTML5 with WebGL could also realize fast $3 \mathrm{D}$ rendering. Figure 4 presents $3 \mathrm{D}$ colon models rendered by Java-, Flash-, and HTML5-based apps. The user could also interact with this model and perform various operations, such as rotation and translation, using the mouse.

Figure 4. Screenshot of a 3D model of the entire colon in the browser. (left) Java, (center) Flash, and (right) HTML5.

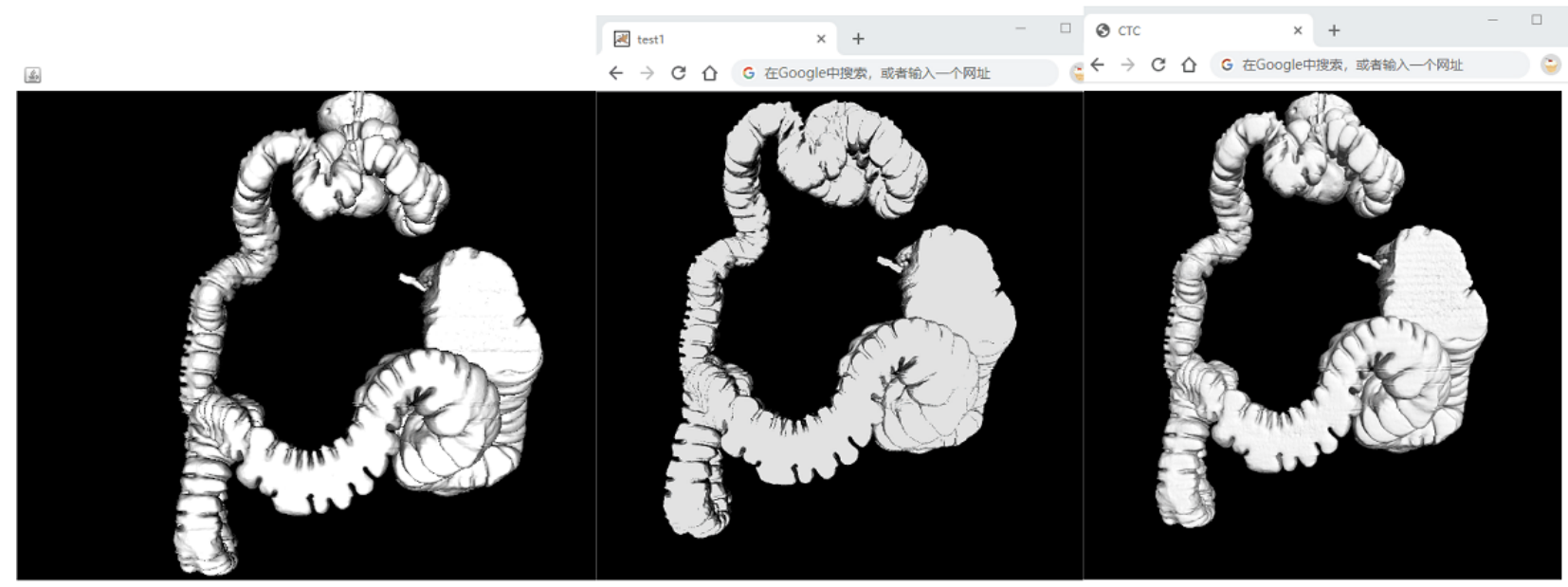

Virtual fly-through navigation is a function used to manipulate the results of $3 \mathrm{D}$ reconstruction. It creates a virtual camera within the colon that moves along a planned path (commonly referred to as the colon centerline [21]); the radiologist can observe the interior of the colon using the continuous movement of the camera. This advanced imaging technique can help the radiologist make more accurate judgments about the lesion. Figure 5 presents the results of different techniques for implementing 3D fly-through within the colon, running in a browser.
Volume rendering is another type of 3D visualization that can represent the interior information of the 3D data set. Our implementation of volume rendering was based on a ray casting algorithm due to its ability to render high quality images [22]. This technique involved intensive computations resulting in low rendering speeds; however, it was feasible to define a subvolume to represent a region of interest. Volume rendering was then applied in this subvolume. In our apps, the size of the subvolume was $100 \times 100 \times 100$ pixels and could be selected by the user. Figure 6 presents a region of interest rendered by the ray casting algorithm in Java-, Flash-, and HTML5-based apps. 
Figure 5. Screenshot of the implementation of the fly-through in the browser. (left) Java, (center) Flash, and (right) HTML5.

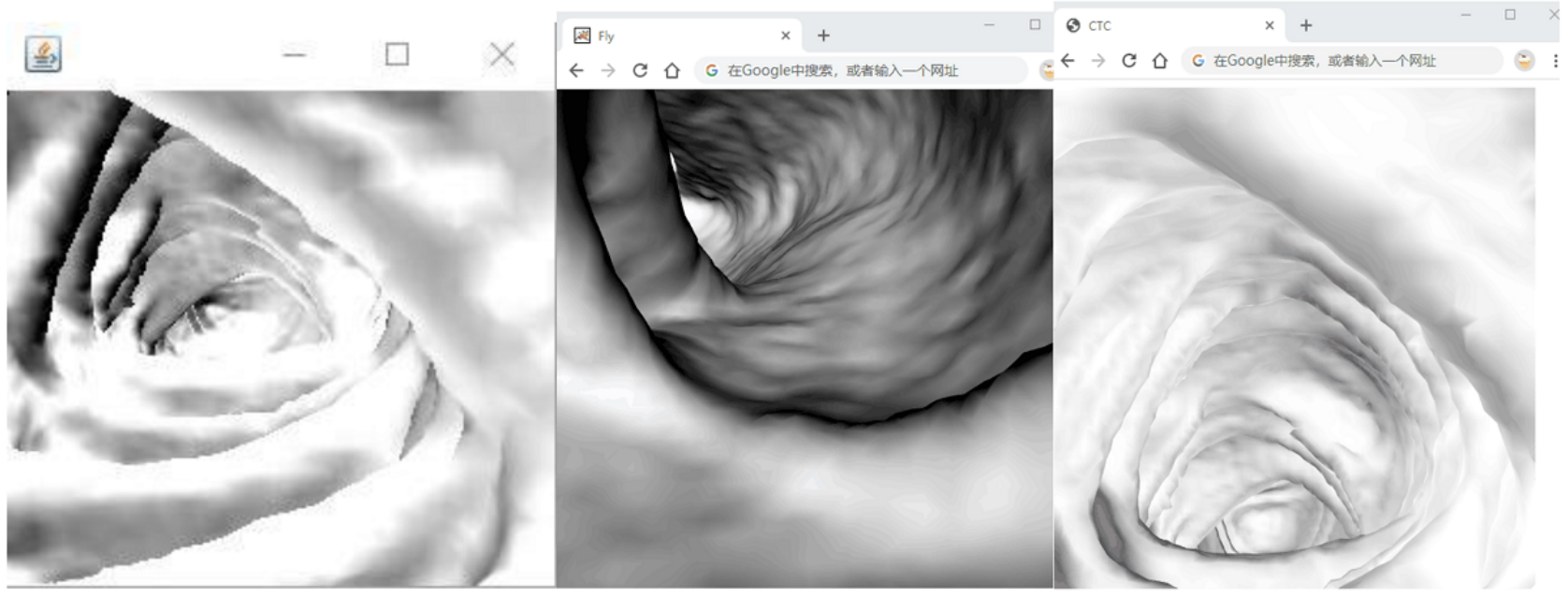

Figure 6. Screenshot of volume rendering in the browser. (left) Java, (center) Flash, and (right) HTML5.

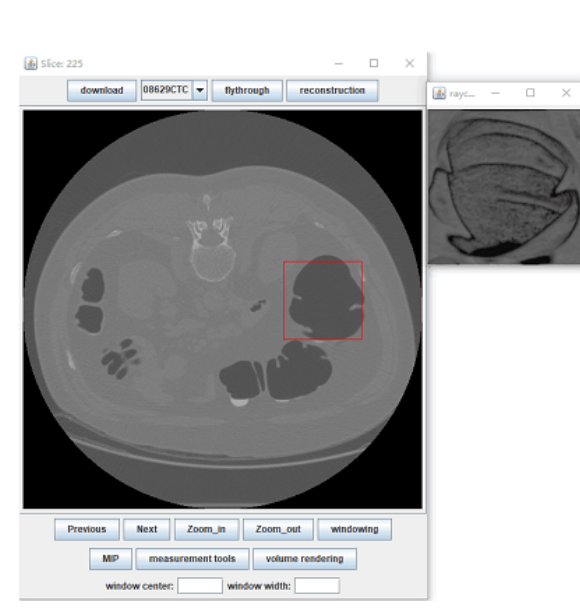

\section{Experiment Design}

\section{Overview}

To compare the performances of the Java-, Flash-, and HTML5-based apps, 3 types of experiments were conducted: determining the performance of the apps running on the same platform (in Windows; Experiment 1); evaluating the performance of the apps on multiple platforms (Experiment 2);

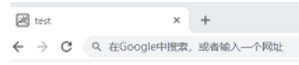
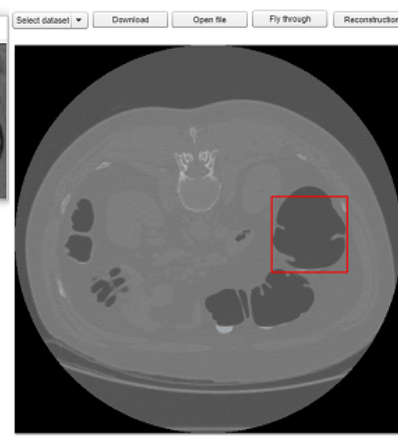

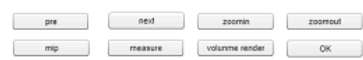

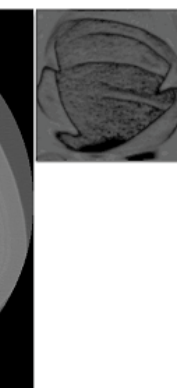

: 圆 $\mathrm{ac}$
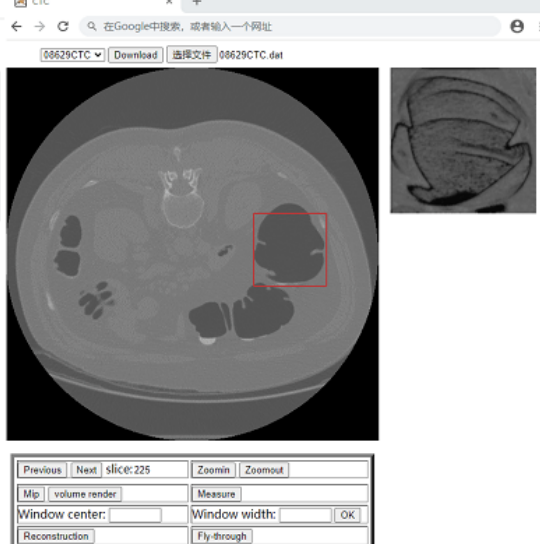

Table 1. CT data sets used in the experiments.

\begin{tabular}{llllll}
\hline Data set & \multicolumn{2}{l}{ Data set size Size } & \multicolumn{2}{l}{ 3D visualization } & \\
& $\mathrm{kB}$ & pixel & Vertex file $(\mathrm{kB})$ & Normal file $(\mathrm{kB})$ & Number of faces \\
\hline 1 & 321,536 & $512 \times 512 \times 628$ & 57,747 & 57,747 & $1,642,580$ \\
2 & 312,320 & $512 \times 512 \times 610$ & 59,545 & 59,545 & $1,693,700$ \\
3 & 256,000 & $512 \times 512 \times 500$ & 60,236 & 60,236 & $1,713,372$ \\
\hline
\end{tabular}

It should be noted that while the performances of Java- and Flash-based apps are browser independent, they are dependent on Java Virtual Machine and Flash Player, respectively. However, HTML5 is solely dependent on the browser, and our previous research [15] has demonstrated that Google Chrome

comparing the performances of the apps using a local area network (LAN) or a wide area network (WAN) (Experiment $3)$. CT colonography data sets $(n=3)$, which were downloaded from The Cancer Imaging Archive [23], were used. Descriptions of the data sets are presented in Table 1, and the information about the computers used in the experiments is provided in Table 2. It is evident that the computers were ordinary personal computers for regular users.

can provide stable and excellent HTML5 performance. Thus, Google Chrome was used to run the HTML5-based app as well as to launch the Flash- and Java-based apps in this experiment. The details of the testing metrics in this study are presented in Table 3. 
Table 2. Computers used in the experiments.

\begin{tabular}{llllll}
\hline Computer & Type & Operating system & CPU & Memory & GPU $^{\mathrm{b}}$ \\
\hline 1 & Desktop & Windows & Intel Core i5-8400 @ 2.80 GHz & $16.00 \mathrm{~GB}$ & $\begin{array}{l}\text { NVIDIA GeForce GTX } \\
1050 \mathrm{Ti}\end{array}$ \\
& Desktop & Ubuntu 20.04 & Intel Core i5-8400 @ 2.80 GHz & $16.00 \mathrm{~GB}$ & $\begin{array}{l}\text { NVIDIA GeForce GTX } \\
1050 \mathrm{Ti}\end{array}$ \\
& & & & Intel Iris Plus Graphics \\
3 & Laptop (Mac- & MacOS Sierra & Intel Core Intel i5 @ 2.30 GHz & $8.00 \mathrm{~GB}$ & 640 \\
\hline
\end{tabular}

${ }^{\mathrm{a}} \mathrm{CPU}$ : central processing unit.

${ }^{\mathrm{b}} \mathrm{GPU}$ : graphics processing unit.

Table 3. Details of testing metrics of this study.

\begin{tabular}{|c|c|c|c|c|c|}
\hline \multirow[t]{2}{*}{ Function } & \multirow[t]{2}{*}{ Label } & \multirow[t]{2}{*}{ Description } & \multicolumn{3}{|c|}{ Measurement } \\
\hline & & & Java & Adobe Flash & HTML5 \\
\hline \multicolumn{6}{|c|}{ Data access } \\
\hline & M1 & Execution time for downloading a medical image data set & Java & ActionScript 3.0 & Manual \\
\hline \multicolumn{6}{|c|}{ 2D image processing } \\
\hline & M2 & Execution time for viewing a slice in a medical image data set & Java & ActionScript 3.0 & JavaScript \\
\hline & M3 & Execution time for implementing windowing per slice & Java & ActionScript 3.0 & JavaScript \\
\hline & M4 & Execution time for implementing magnification per slice & Java & ActionScript 3.0 & JavaScript \\
\hline & M5 & Execution time for implementing mouse wheel per slice & Java & ActionScript 3.0 & JavaScript \\
\hline & M6 & Execution time for implementing measure tool per slice & Java & ActionScript 3.0 & JavaScript \\
\hline & M7 & Execution time for implementing maximum intensity projection & Java & ActionScript 3.0 & JavaScript \\
\hline \multicolumn{6}{|c|}{ 3D visualization } \\
\hline & M8 & $\begin{array}{l}\text { Execution time for downloading the vertex and normal files of } \\
\text { a medical image data set }\end{array}$ & Java & ActionScript 3.0 & Manual \\
\hline & M9 & $\begin{array}{l}\text { Execution time for rendering a 3D model based on the download- } \\
\text { ed vertex and normal files }\end{array}$ & Java & ActionScript 3.0 & JavaScript \\
\hline & M10 & Frame rate of fly-through & Java & ActionScript 3.0 & JavaScript \\
\hline & M11 & Frame rate of software-based volume rendering & Java & ActionScript 3.0 & JavaScript \\
\hline
\end{tabular}

\section{Experiment 1}

The first experiment was carried out with 3 data sets using LAN to compare the performances of different apps running on the same platform. Computer 1 was chosen to test the Java-, Flash-, and HTML5-based apps on a Windows operating system. Each function was implemented 20 times in each app.

\section{Experiment 2}

The second experiment was used to determine the performance consistency of different internet technologies among multiple platforms. Computers 1, 2, and 3 were used in this experiment. Therefore, Java-, Flash-, and HTML5-based apps were run on Windows, macOS, and Linux platforms, respectively. Each function in the 3 apps was implemented 20 times on these platforms using data set 1 over a LAN.

\section{Experiment 3}

In the third experiment, computer 1 was used to evaluate the performances of Java-, Flash-, and HTML5-based apps over the LAN and WAN. This computer was equipped with
Windows, and hence all the 3 apps were tested on the same platform. All 3 data sets were used in the experiment. Java-, Flash-, and HTML5-based apps were tested based on data sets 1,2 , and 3 , to determine the performance differences when they ran over a LAN and WAN. In WAN, the 3 apps accessed the medical data set and vertex and normal files on the remote server. The bandwidth of the connecting network was $50 \mathrm{Mbps}$, and it had a download speed of approximately $5.1 \mathrm{MB} / \mathrm{s}$. The download sizes for the medical image data set, vertex file, and normal file are listed in Table 2.

Each function in the app was implemented 20 times by Java Virtual Machine (version 1.8.0), Flash Player (version 32.0.0.433), and Chrome (version 83.0.4103.97), either over the LAN or WAN.

\section{Radiologist Feedback}

In order to collect feedback on the apps, we conducted a pilot trial. Radiologists $(n=5)$ at Wuhan Hospital of Traditional Chinese Medicine participated in this trial. They first received a brief introduction of the project and our medical imaging apps. 
After that, each of them was required to interpret $10 \mathrm{CT}$ examinations using the Java-based app, $10 \mathrm{CT}$ examinations using the Flash-based app, and 10 CT examinations using the HTML5-based apps on a Windows personal computer on the LAN.

After the trial, they filled out questionnaires (Multimedia Appendix 1). A 5-point Likert scale was used to represent the radiologists' opinions on a particular question or statement: strongly disagree, disagree, unsure, agree, and strongly agree. Radiologists' responses were recorded on a 1-5 scale, with higher numbers representing stronger agreement.

\section{Results}

\section{Experiment 1: General Performance}

The average performances for each function are presented in Table 4 (Experiment 1). The comparison revealed that each technology had its own advantages. Java was associated with the shortest downloading time and highest frame rate for software-based volume rendering. However, it performed poorly at surface rendering. HTML5 surface rendering performed best. In terms of 2D functions, such as zooming and windowing, all 3 apps performed similarly. Overall, HTML5 outperformed the other 2 technologies, with the exception of downloading and software-based volume rendering. 
Table 4. Comparison of the performances of the 3 apps in various scenarios.

\begin{tabular}{|c|c|c|c|c|c|c|c|c|c|c|c|}
\hline \multirow[t]{2}{*}{ Technology } & \multirow{2}{*}{$\begin{array}{l}\text { Data access } \\
\text { M1 }\left(\mathrm{s}^{\mathrm{a}}\right)\end{array}$} & \multicolumn{4}{|c|}{ 2D image processing } & \multirow[b]{2}{*}{ M6 (s) } & \multirow[b]{2}{*}{ M7 (s) } & \multicolumn{2}{|c|}{$3 \mathrm{D}$ visualization } & \multirow[b]{2}{*}{$\begin{array}{l}\text { M10 } \\
\left(\mathrm{fps}^{\mathrm{b}}\right)\end{array}$} & \multirow[b]{2}{*}{$\begin{array}{l}\text { M11 } \\
\text { (fps) }\end{array}$} \\
\hline & & M2 (s) & M3 (s) & M4 (s) & M5 (s) & & & M8 (s) & M9 (s) & & \\
\hline \multicolumn{12}{|l|}{ Experiment 1} \\
\hline \multicolumn{12}{|l|}{ Data set 1} \\
\hline $\begin{array}{l}\text { Java (Win- } \\
\text { dows) }\end{array}$ & 29.87 & 0.0073 & 0.0142 & 0.0015 & 0.0077 & 0.0010 & 0.3446 & 10.76 & 271.21 & 32.25 & 1.92 \\
\hline $\begin{array}{l}\text { Flash } \\
\text { (Windows) }\end{array}$ & 27.76 & 0.0144 & 0.0193 & 0.0004 & 0.0146 & 0.0001 & 26.7071 & 10.24 & 16.20 & 10.21 & 0.05 \\
\hline $\begin{array}{l}\text { HTML5 } \\
\text { (Windows) }\end{array}$ & 28.58 & 0.0009 & 0.0133 & 0.0006 & 0.0008 & 0.0002 & 2.4036 & 13.46 & 1.09 & 59.30 & 0.56 \\
\hline \multicolumn{12}{|l|}{ Data set 2} \\
\hline $\begin{array}{l}\text { Java (Win- } \\
\text { dows) }\end{array}$ & 29.16 & 0.0066 & 0.0094 & 0.0016 & 0.0058 & 0.0010 & 0.3701 & 10.29 & 280.54 & 34.23 & 1.97 \\
\hline $\begin{array}{l}\text { Flash } \\
\text { (Windows) }\end{array}$ & 26.97 & 0.0148 & 0.0189 & 0.0007 & 0.0149 & 0.0002 & 27.5514 & 10.35 & 17.59 & 9.91 & 0.05 \\
\hline $\begin{array}{l}\text { HTML5 } \\
\text { (Windows) }\end{array}$ & 27.98 & 0.0008 & 0.0154 & 0.0004 & 0.0009 & 0.0003 & 2.4560 & 12.38 & 1.25 & 60.05 & 0.61 \\
\hline \multicolumn{12}{|l|}{ Data set 3} \\
\hline $\begin{array}{l}\text { Java (Win- } \\
\text { dows) }\end{array}$ & 23.96 & 0.0046 & 0.0098 & 0.0018 & 0.0049 & 0.0008 & 0.2923 & 11.13 & 283.79 & 36.25 & 2.24 \\
\hline $\begin{array}{l}\text { Flash } \\
\text { (Windows) }\end{array}$ & 22.11 & 0.0142 & 0.0186 & 0.0005 & 0.0144 & 0.0002 & 21.1775 & 10.42 & 18.72 & 10.13 & 0.06 \\
\hline $\begin{array}{l}\text { HTML5 } \\
\text { (Windows) }\end{array}$ & 23.19 & 0.0008 & 0.0151 & 0.0005 & 0.0008 & 0.0002 & 1.9772 & 12.37 & 1.43 & 60.10 & 0.71 \\
\hline \multicolumn{12}{|l|}{ Experiment 2} \\
\hline \multicolumn{12}{|l|}{ Data set 1} \\
\hline $\begin{array}{l}\text { Java (Win- } \\
\text { dows) }\end{array}$ & 29.87 & 0.0073 & 0.0142 & 0.0015 & 0.0077 & 0.0010 & 0.3446 & 10.76 & 271.21 & 32.25 & 1.92 \\
\hline $\begin{array}{l}\text { Flash } \\
\text { (Windows) }\end{array}$ & 27.76 & 0.0144 & 0.0193 & 0.0004 & 0.0146 & 0.0001 & 26.7071 & 10.24 & 16.20 & 10.21 & 0.05 \\
\hline $\begin{array}{l}\text { HTML5 } \\
\text { (Windows) }\end{array}$ & 28.58 & 0.0009 & 0.0133 & 0.0006 & 0.0008 & 0.0002 & 2.4036 & 13.46 & 1.09 & 59.30 & 0.56 \\
\hline \multicolumn{12}{|l|}{ Data set 1} \\
\hline $\begin{array}{l}\text { Java (ma- } \\
\text { cOS) }\end{array}$ & 28.93 & 0.0132 & 0.0189 & 0.0028 & 0.0133 & 0.0002 & 0.3483 & 10.27 & $\underline{c}^{\mathrm{c}}$ & - & 1.42 \\
\hline $\begin{array}{l}\text { Flash (ma- } \\
\text { cOS) }\end{array}$ & 27.76 & 0.0207 & 0.0232 & 0.0002 & 0.0191 & 0.0006 & 21.4324 & 9.98 & 13.59 & 10.07 & 0.07 \\
\hline $\begin{array}{l}\text { HTML5 } \\
\text { (macOS) }\end{array}$ & 29.04 & 0.0011 & 0.0194 & 0.0009 & 0.0012 & 0.0002 & 2.6352 & 12.31 & 0.9285 & 60.20 & 0.54 \\
\hline \multicolumn{12}{|l|}{ Data set 1} \\
\hline $\begin{array}{l}\text { Java (Lin- } \\
\text { ux) }\end{array}$ & 19.61 & 00113 & 0.0177 & 0.0052 & 0.0104 & 0.0002 & 0.4398 & 8.49 & 77.84 & 19.33 & 1.68 \\
\hline $\begin{array}{l}\text { Flash (Lin- } \\
\text { ux) }\end{array}$ & 27.75 & 0.0291 & 0.0513 & 0.0003 & 0.0325 & 0.0004 & 16.3715 & 9.98 & 15.12 & 9.26 & 0.11 \\
\hline $\begin{array}{l}\text { HTML5 } \\
\text { (Linux) }\end{array}$ & 33.22 & 0.0021 & 0.0414 & 0.0008 & 0.0019 & 0.0004 & 3.7369 & 13.95 & 1.39 & 58.7 & 0.37 \\
\hline \multicolumn{12}{|l|}{ Experiment 3} \\
\hline Data set 1 & & & & & & & & & & & \\
\hline
\end{tabular}




\begin{tabular}{|c|c|c|c|c|c|c|c|c|c|c|c|}
\hline \multirow[t]{2}{*}{ Technology } & \multirow{2}{*}{$\begin{array}{l}\text { Data access } \\
\text { M1 }\left(\mathrm{s}^{\mathrm{a}}\right)\end{array}$} & \multicolumn{6}{|c|}{ 2D image processing } & \multicolumn{4}{|c|}{$3 \mathrm{D}$ visualization } \\
\hline & & M2 (s) & M3 (s) & M4 (s) & M5 (s) & M6 (s) & M7 (s) & M8 (s) & M9 (s) & $\begin{array}{l}\text { M10 } \\
\left(\mathrm{fps}^{\mathrm{b}}\right)\end{array}$ & $\begin{array}{l}\text { M11 } \\
\text { (fps) }\end{array}$ \\
\hline $\begin{array}{l}\text { Java } \\
\text { (LAN) }\end{array}$ & 29.87 & 0.0073 & 0.0142 & 0.0015 & 0.0077 & 0.0010 & 0.3446 & 10.76 & 271.21 & 32.25 & 1.92 \\
\hline $\begin{array}{l}\text { Java } \\
\text { (WAN) }\end{array}$ & 311.47 & 0.0071 & 0.0153 & 0.0011 & 0.0072 & 0.0010 & 0.3417 & 169.89 & 268.65 & 32.23 & 1.89 \\
\hline \multicolumn{12}{|l|}{ Data set 2} \\
\hline $\begin{array}{l}\text { Flash } \\
\text { (LAN) }\end{array}$ & 26.97 & 0.0148 & 0.0189 & 0.0007 & 0.0149 & 0.0002 & 27.5514 & 10.35 & 17.59 & 9.91 & 0.05 \\
\hline $\begin{array}{l}\text { Flash } \\
\text { (WAN) }\end{array}$ & 464.05 & 0.0145 & 0.0191 & 0.0006 & 0.0148 & 0.0003 & 27.4085 & 176.42 & 69.18 & 9.17 & 0.05 \\
\hline \multicolumn{12}{|l|}{ Data set 3} \\
\hline $\begin{array}{l}\text { HTML5 } \\
\text { (LAN) }\end{array}$ & 23.19 & 0.0008 & 0.0151 & 0.0005 & 0.0008 & 0.0002 & 1.9772 & 12.37 & 1.43 & 60.10 & 0.71 \\
\hline $\begin{array}{l}\text { HTML5 } \\
\text { (WAN) }\end{array}$ & 377.51 & 0.0009 & 0.0162 & 0.0008 & 0.0008 & 0.0006 & 2.0147 & 186.39 & 1.31 & 60.02 & 0.72 \\
\hline
\end{tabular}

$\mathrm{a}_{\mathrm{s}}$ : seconds.

$\mathrm{b}_{\text {fps: frames per second. }}$

${ }^{\mathrm{c}}$ Not tested in macOS.

\section{Experiment 2: Performance on Multiple Platforms}

The average performances of all the functions are presented in Table 4 (Experiment 2). It can be observed from this table that although the 2D performances of Java-, Flash-, and HTML5-based apps running on multiple platforms (Windows, macOS, and Linux) were almost the same, there are obvious differences in $3 \mathrm{D}$ performance. Owing to the facts that Java3D is obsolete and the configuration in macOS was much more complicated than expected, surface rendering by Java was not tested in macOS but only tested in Windows and Linux. In terms of the 3D performance on different platforms, Java-based apps on Windows achieved better performance than on Linux and macOS. However, Flash- and HTML5-based apps demonstrated consistent performance across different platforms.

\section{Experiment 3: Performance based on LAN and WAN}

The average performances for each function are presented in Table 4 (Experiment 3). The results of the performances of Java-, Flash-, and HTML5-based apps over LAN and WAN revealed that there was little difference between the LAN and WAN, except for downloading. Given that the data transmission speed over the WAN was lower than that over the LAN, the downloading time was different, as expected. After downloading data to the client computer, the app performance over the WAN was the same as that over the LAN.

\section{Summary}

The experimental results demonstrated that Java-, Flash-, and HTML5-based apps have the ability to yield real-time performances for all 2D functions. However, the 3D performances differed between the 3 apps. In terms of software-based volume rendering, the Java-based app had the highest frame rate; however, it required the longest amount of time for surface rendering and failed to run surface rendering in macOS. In terms of surface rendering, the HTML5-based app had the fastest rendering and the highest speed for fly-through without platform dependence. However, the frame rate of software-based volume rendering by HTML5 was slightly lower than that by Java. The 3D performances of the Flash-based app were worse than both of the other apps.

\section{Pilot Trial and Feedback From Radiologists}

The results of radiologists' responses are presented in Table 5.

Most radiologists were satisfied with the functions that we provided. However, they were not satisfied with 3D functions in Java and Flash. Three radiologists reported that Java took a long time for surface rendering and Flash provided a significantly low frame rate for volume rendering. For question 8 , every radiologist chose HTML5, which means that HTML5 obtained the highest satisfaction among these 3 technologies. 
Table 5. Radiologists' responses to the questionnaires.

\begin{tabular}{|c|c|c|c|c|c|c|c|c|}
\hline \multirow[t]{2}{*}{ Radiologist } & \multicolumn{8}{|c|}{ Question score $^{\mathrm{a}}$} \\
\hline & 1 & 2 & 3 & 4 & 5 & 6 & 7 & $8^{\mathrm{b}}$ \\
\hline Radiologist 1 & 5 & 5 & 5 & 5 & 5 & 5 & 5 & 3 \\
\hline Radiologist 2 & 4 & 5 & 5 & 5 & 3 & 3 & 4 & 3 \\
\hline Radiologist 3 & 5 & 5 & 5 & 5 & 4 & 4 & 5 & 3 \\
\hline Radiologist 4 & 5 & 5 & 5 & 5 & 4 & 4 & 5 & 3 \\
\hline Radiologist 5 & 5 & 5 & 5 & 5 & 5 & 5 & 5 & 3 \\
\hline Total score & 24 & 25 & 25 & 25 & 21 & 21 & 24 & $\mathrm{~N} / \mathrm{A}^{\mathrm{c}}$ \\
\hline
\end{tabular}

${ }^{\mathrm{a}}$ From 1 (strongly disagree) to 5 (strongly agree).

boptions: 1 (Java), 2 (Flash), 3 (HTML5).

${ }^{\mathrm{c}} \mathrm{N} / \mathrm{A}$ : not applicable.

\section{Discussion}

\section{Principal Findings}

Currently, there are 3 main technologies for the development of web-based medical imaging apps, namely, Oracle Java, Adobe Flash, and HTML5. Around the 2000s, Oracle Java was a popular internet technology in the field of medical imaging [7-12,14,24]. Since 2010, Flash-based imaging apps have appeared, owing to the ubiquity and small size of the Flash Player $[13,16]$. Since the release of the World Wide Web Consortium HTML5 recommendation in 2014, there has been a growing trend toward the utilization of HTML5 in the development of medical imaging apps. McLaughlin et al [25] developed a digital training platform for interpreting radiographic images based on HTML5. Their platform had 2 tools, a search strategy training tool and an eye tracking tool, which were used to clarify the image interpretation process [25]. Gorgbjerg [26] presented an HTML5-based web app that could be manipulated as in a picture archiving and communication systems. Zhang [27] created a network-based medical data rendering and sharing system with a client app that was developed by HTML5. This client app has the ability to deliver real-time visualization on the web. Additionally, our previous study [15] provided an evaluation of HTML5 for medical imaging apps and demonstrated that HTML5 can provide an excellent remote access medical imaging experience.

In this study, 3 technologies were used to develop a comprehensive medical imaging app and to evaluate the performances of these technologies in the field of radiology. In terms of accessibility, both Java- and Flash-based apps require a browser plug-in. Despite the fact that the Flash Player has long been one of the most popular browser plug-ins, Apple decided to stop bundling Flash Player in macOS in 2010. Thus, for this group of users, to be able to run Flash-based apps, they must initially install Flash Player. Similarly, to be able to run Java, Java Virtual Machine must be installed. HTML5 does not suffer from this problem because it is the native language used in all browsers. Therefore, HTML5 requires no preinstallation and is a platform-independent technology that provides a high level of accessibility. However, the advantages associated with HTML5 exist only in the latest version of browsers. Older browser versions, such as Microsoft Internet Explorer 8 (or older versions), Mozilla Firefox 3.5 (or older versions), and Google Chrome 10 (or older versions), are not compatible with HTML5. In these cases, users would be required to update their browsers, otherwise, HTML5-based apps could not be launched in their browsers. Furthermore, browsers vary in their level of support for the HTML5 standard, and thus, this leads to inconsistent user experiences. For example, the implementation of the mouse wheel event is different among Internet Explorer, Chrome, Safari, and Firefox. In the case of Chrome, when the mouse wheel is rolled up, the value increases however, in Firefox, the value decreases.

In terms of functionality, all 3 technologies can realize the necessary functions for remote viewing of radiological images. Image processing, such as zooming and windowing, can be provided by all 3 technologies on all platforms. However, implementation of $3 \mathrm{D}$ visualization is more complicated than the implementation of image processing, especially for Oracle Java. Oracle Java realizes 3D surface rendering by depending on Java3D API. However, this API has not been updated since 2008. Hence, some problems emerge in recent implementations of Java3D (eg, the Java3D app failed to launch in macOS). 3D visualization by Adobe Flash and HTML5 can be successfully implemented, regardless of the platform. However, it should be noted that Adobe will terminate its support for Flash at the end of 2020. Thereafter, Flash 3D API and Flash Player will not be updated. In this case, only HTML5 has an advanced API and hence can provide a higher level of functionality (compared to Oracle Java and Adobe Flash).

In terms of usability, the experimental results reveal that all 3 technologies can provide 2D image processing on all platforms. However, the 3D performances of these technologies are different. Among these technologies, HTML5 presents the best surface rendering performances in terms of rendering time and frame rate. In terms of volume rendering, HTML5 is not good at software-based volume rendering. However, when integrated with a GPU, HTML5 can provide fast hardware-based volume rendering $[28,29]$.

In terms of interoperability, Oracle Java, Adobe Flash, and HTML5 are designed for developing rich web apps. Therefore, all 3 apps can be connected to a large teleradiology system and 
placed as client components. Moreover, the source code of HTML5 is exposed online, and therefore, the locations of image data sets can be easily assessed. The source codes of Java and Flash are hidden (inside .JAR and .SWF files, respectively), which prevents unauthorized access to image data sets. Thus, Java and Flash outperform HTML5 in data privacy.

Recently, cloud computing has been used in the field of medical imaging for high-capacity storage, sharing, and intensive computational tasks $[30,31]$. In this infrastructure, the image data and complex processing tasks are moved from user computers to the cloud. The users then launch an app to access the cloud. In this case, a radiologist can implement the cloud-based medical image analysis using a personal computer from any location. Furthermore, web technology supports the development of the client app in the cloud-based system. With its development, the client app can become more powerful than before. Among these web technologies, HTML5 can develop a zero-footprint web viewer, which requires zero plug-ins, zero latency, and zero maintenance. Therefore, most commercially web-based DICOM (Digital Imaging and Communications in Medicine standard) viewers, such as Ambra [32], medDream [33], and boxDicom [34], switched to an HTML5-based solution recently. All of them can be integrated into any picture archiving and communication systems system. Additionally, medDream provides 3D features, such as maximum intensity projection and 3D rendering, in a browser. We confirmed that their HTML5 solutions can implement necessary interpretation tools, such as 2D image processing and 3D visualization, inside the client browser with satisfactory performance.

Although web technology enables remote viewing of radiological images easily and efficiently, there are still 4 issues affecting current web-based medical imaging apps. First, when data are transmitted over the internet, security is the biggest challenge, and this has encouraged many researches to find ways to keep medical images safe and confidential $[35,36]$. Second, remote viewing of radiological images is heavily dependent on the network. When internet connections are slow or unavailable, our web apps cannot work properly; hence, network condition is an important factor in teleradiology settings. Third, the specifications of a personal computer are usually inferior to those of dedicated workstations, and therefore, intensive computational tasks, such as volume rendering, cannot be implemented on a personal computer. In our implementation of volume rendering, the rendering region was reduced in order to provide fast volume rendering. Therefore, some interpretation tools need to be customized and simplified in web-based apps. Furthermore, typical medical image sizes range from $512 \times 512 \times 8$ bits up to $1024 \times 1024 \times 12$ bits. For some imaging apps, the resolution is even higher. Therefore, the client's screen should support higher resolutions, otherwise the medical images cannot be properly displayed.

\section{Limitations}

Our study and the web-based apps that were developed also have some limitations. First, we were able to obtain feedback from 5 radiologists to conduct a pilot testing, but we were not able to conduct a large and comprehensive investigation on users' opinions. Therefore, the feedback of users may contain deviations due to a small sample size. Second, only 2 personal computers, one on which Windows and Linux were installed, and another on which macOS was installed, were used in our experiments. The performances of apps may be affected by the hardware specifications. In future, upgraded computer hardware could enhance the performance of our apps.

\section{Conclusion}

Based on the review of existing literature, it is apparent that there is a lack of studies on the evaluation of different internet technologies for remote viewing of radiological images. In this study, 3 main internet technologies (ie, Oracle Java, Adobe Flash, and HTML5) were used to develop comprehensive web-based medical imaging apps. Experiments were conducted to compare these technologies in terms of accessibility, functionality, and usability. Moreover, advantages and disadvantages were discussed. Our research demonstrated that HTML5 is a promising technology for remote viewing of radiological images and can provide excellent performance without requiring any plug-ins. Therefore, our research provides an important reference for future development of web apps in the field of medical imaging, and it could help to identify an optimal solution for remote viewing of radiological images.

\section{Acknowledgments}

This work was supported by the self-determined research funds of Central China Normal University from the colleges' basic research and operation of Ministry of Education (No. CCNU20QN019).

\section{Authors' Contributions}

All authors contributed to the design of the study. BH and LX designed the medical imaging apps; QM and XW collected the data sets and developed the Java-, Flash-, and HTML5-based apps; all authors performed the experiments. QM wrote the main body of the manuscript, and all authors reviewed the manuscript.

\section{Conflicts of Interest}

None declared.

\section{Multimedia Appendix 1}

Questionnaire. 


\section{References}

1. James J, Davies A, Cowen A, O'Connor P. Developments in digital radiography: an equipment update. Eur Radiol 2001 Mar 13;11(12):2616-2626. [doi: $10.1007 / \mathrm{s} 003300100828$ ] [Medline: $\underline{11734969]}$

2. Bowers GH, Steiner E, Kalman M. Implementing teleradiology in a private radiology practice: lessons learned. J Digit Imaging 1998 Aug;11(3 Suppl 1):96-98 [FREE Full text] [doi: 10.1007/BF03168271] [Medline: 9735443]

3. Fraternali P, Rossi G, Sánchez-Figueroa F. Rich internet application. IEEE Internet Comput 2010 May;14(3):9-12. [doi: 10.1109/mic.2010.76]

4. JDK 12 documentation. Oracle. URL: https://docs.oracle.com/en/java/javase/12/index.html [accessed 2019-07-05]

5. ActionScript 3.0. Reference for the Adobe Flash Platform. 2019. URL: https://help.adobe.com/en US/FlashPlatform/ reference/actionscript/3/ [accessed 2019-07-05]

6. Living standard. HTML. URL: http://w3c.github.io/html/ [accessed 2019-07-10]

7. Slomka PJ, Elliott E, Driedger AA. Java-based remote viewing and processing of nuclear medicine images: toward "the imaging department without walls". J Nucl Med 2000 Jan;41(1):111-118. [Medline: 10647613]

8. Knoll P, Höll K, Mirzaei S, Koriska K, Köhn H. Distributed nuclear medicine applications using World Wide Web and Java technology. Eur Radiol 2000 Aug 18;10(9):1483-1486. [doi: 10.1007/s003300000432] [Medline: 10997440]

9. Choi H, Park S, Kang J, Kim S, Choi H. Tele-medical imaging conference system based on the Web. Comput Methods Programs Biomed 2002 Jun;68(3):223-231. [doi: 10.1016/s0169-2607(01)00174-2] [Medline: 12074849]

10. Kamauu AWC, DuVall SL, Wiggins RH, Avrin DE. Using applet-servlet communication for optimizing window, level and crop for DICOM to JPEG conversion. J Digit Imaging 2008 Sep;21(3):348-354 [FREE Full text] [doi: 10.1007/s10278-007-9038-3] [Medline: 17534682]

11. Min Q, Sadleir R. Distributed medical imaging apps using Java technology. 2015 Dec 16 Presented at: International Congress on Image and Signal Processing BioMedical Engineering and Informatics (CISP-BMEI); 2015; Hong Kong, China p. 16-19. [doi: 10.1109/APSIPA.2015.7415343]

12. Looney PT, Young KC, Halling-Brown MD. MedXViewer: providing a web-enabled workstation environment for collaborative and remote medical imaging viewing, perception studies and reader training. Radiat Prot Dosimetry 2016 Jun;169(1-4):32-37. [doi: 10.1093/rpd/ncv482] [Medline: 26628613]

13. Min Q, Wang Z, Wu M. An evaluation of adobe flash for remote access medical imaging applications. 2016 Oct 15 Presented at: IEEE 9th International Congress on Image and Signal Processing, BioMedical Engineering and Informatics (CISP-BMEI); 2016; Datong, China p. 15-17. [doi: 10.1109/cisp-bmei.2016.7852789]

14. Doel T, Shakir DI, Pratt R, Aertsen M, Moggridge J, Bellon E, et al. GIFT-Cloud: A data sharing and collaboration platform for medical imaging research. Comput Methods Programs Biomed 2017 Feb;139:181-190 [FREE Full text] [doi: $\underline{10.1016 / j . c m p b .2016 .11 .004] ~[M e d l i n e: ~ 28187889] ~}$

15. Min Q, Wang Z, Liu N. An evaluation of HTML5 and WebGL for medical imaging applications. J Healthc Eng 2018 Aug 29;2018:1592821 [FREE Full text] [doi: 10.1155/2018/1592821] [Medline: 30245782]

16. Arguinarena EJC, Macchi JE, Escobar P, Del Fresno M, Massa J, Santiago M. Dcm-ar: a fast flash-based Web-PACS viewer for displaying large DICOM images. In: Conf Proc IEEE Eng Med Biol Soc. 2010 Presented at: International Conference of the IEEE Engineering in Medicine and Biology; Aug 31-Sept 4; Buenos Aires, Argentina p. 3463-3466. [doi: 10.1109/IEMBS.2010.5627827]

17. Rosenkrantz AB, Hanna TN, Steenburg SD, Tarrant MJ, Pyatt RS, Friedberg EB. The current state of teleradiology across the United States: a national survey of radiologists' habits, attitudes, and perceptions on teleradiology practice. J Am Coll Radiol 2019 Dec;16(12):1677-1687. [doi: 10.1016/j.jacr.2019.05.053] [Medline: 31271736]

18. Haj-Mirzaian A, Sethi N, de Francesca B, Sahni S, Zaheer A. Web-based radiology subspecialty training program: pilot feasibility and effectiveness analysis on Ethiopian radiologists. Acad Radiol 2020 Feb;27(2):293-299. [doi: 10.1016/j.acra.2019.02.025] [Medline: $\underline{31060981]}$

19. Pickhardt PJ, Lee AD, Taylor AJ, Michel SJ, Winter TC, Shadid A, et al. Primary 2D versus primary 3D polyp detection at screening CT colonography. AJR Am J Roentgenol 2007 Dec;189(6):1451-1456. [doi: 10.2214/AJR.07.2291] [Medline: $\underline{18029884]}$

20. Lorensen W, Cline H. Marching cubes: a high resolution 3D surface construction algorithm. 1987 Presented at: SIGGRAPH '87: Proceedings of the 14th Annual Conference on Computer Graphics and Interactive Techniques; August 1987; New York, NY, USA. [doi: 10.1145/37401.37422]

21. Sadleir RJ, Whelan PF. Fast colon centreline calculation using optimised 3D topological thinning. Comput Med Imaging Graph 2005 Jun;29(4):251-258. [doi: 10.1016/j.compmedimag.2004.10.002] [Medline: 15890252]

22. Levoy M. Display of surfaces from volume data. IEEE Comput Grap Appl 1988 May;8(3):29-37. [doi: 10.1109/38.511]

23. Clark K, Vendt B, Smith K, Freymann J, Kirby J, Koppel P, et al. The Cancer Imaging Archive (TCIA): maintaining and operating a public information repository. J Digit Imaging 2013 Dec 25;26(6):1045-1057 [FREE Full text] [doi: 10.1007/s10278-013-9622-7] [Medline: 23884657] 
24. Poliakov AV, Albright E, Hinshaw KP, Corina DP, Ojemann G, Martin RF, et al. Server-based approach to web visualization of integrated three-dimensional brain imaging data. J Am Med Inform Assoc 2005;12(2):140-151 [FREE Full text] [doi: 10.1197/jamia.M1671] [Medline: 15561787]

25. McLaughlin L, Woznitza N, Cairns A, McFadden S, Bond R, Hughes C, et al. Digital training platform for interpreting radiographic images of the chest. Radiography (Lond) 2018 May;24(2):159-164. [doi: 10.1016/j.radi.2017.12.010] [Medline: 29605114]

26. Borgbjerg J. MULRECON: a web-based imaging viewer for visualization of volumetric images. Curr Probl Diagn Radiol 2019;48(6):531-534. [doi: 10.1067/j.cpradiol.2018.09.001] [Medline: 30340913]

27. Zhang Q. Web-based medical data visualization and information sharing towards application in distributed diagnosis. Inform Med Unlocked 2019;14:69-81. [doi: 10.1016/j.imu.2018.10.010]

28. Arbelaiz A. Volume visualization tools for medical applications in ubiquitous platforms. In: Lecture Notes of the Institute for Computer Sciences, Social Informatics and Telecommunications Engineering.: Springer, Cham; 2017 Presented at: International Summit on eHealth; June 14-16, 2016; Budapest, Hungary p. 443-450. [doi: 10.1007/978-3-319-49655-9_54]

29. Lesar Ž, Bohak C, Marolt M. Real-time interactive platform-agnostic volumetric path tracing in webGL 2.0. 2018 Jun 20 Presented at: Web3D '18: Proceedings of the 23rd International ACM Conference on 3D Web Technology; 2018; Poznań, Poland p. 1-7. [doi: 10.1145/3208806.3208814]

30. Kagadis GC, Kloukinas C, Moore K, Philbin J, Papadimitroulas P, Alexakos C, et al. Cloud computing in medical imaging. Med Phys 2013 Jul 21;40(7):070901. [doi: 10.1118/1.4811272] [Medline: 23822402]

31. Liu L, Chen W, Nie M, Zhang F, Wang Y, He A, et al. iMAGE cloud: medical image processing as a service for regional healthcare in a hybrid cloud environment. Environ Health Prev Med 2016 Nov 25;21(6):563-571 [FREE Full text] [doi: 10.1007/s12199-016-0582-7] [Medline: 27783315]

32. DICOM web viewer: vew from anywhere, anytime, across all imaging types. Ambra Health. URL: https://ambrahealth. com/products-and-services/dicom-web-viewer/ [accessed 2020-09-01]

33. HTML 5 zero-footprint DICOM viewer. MedDream. URL: https://www.softneta.com/products/meddream-dicom-viewer/ [accessed 2020-09-01]

34. Medical images for the cloud. BoxDicom. URL: https://boxdicom.com/ [accessed 2020-09-01]

35. Rodrigues JJPC, de la Torre I, Fernández G, López-Coronado M. Analysis of the security and privacy requirements of cloud-based electronic health records systems. J Med Internet Res 2013 Aug 21;15(8):e186 [FREE Full text] [doi:

10.2196/jmir.2494] [Medline: 23965254]

36. Blanquer I, Brasileiro F, Brito A, Calatrava A, Carvalho A, Fetzer C, et al. Federated and secure cloud services for building medical image classifiers on an intercontinental infrastructure. Future Generation Computer Systems 2020 Sep;110:119-134. [doi: 10.1016/j.future.2020.04.012]

\author{
Abbreviations \\ CT: computed tomography \\ DICOM: Digital Imaging and Communications in Medicine standard \\ GPU: graphics processing unit \\ HTML: hypertext markup language \\ LAN: local area network \\ WAN: wide area network
}

Edited by G Eysenbach; submitted 18.09.19; peer-reviewed by J Borgbjerg, A Korchi, P Lei, J Lee; comments to author 11.03.20;
revised version received 21.07.20; accepted 11.08.20; published 25.09.20
Please cite as:
Min Q, Wang X, Huang B, Xu L
Web-Based Technology for Remote Viewing of Radiological Images: App Validation
J Med Internet Res 2020;22(9):e16224
URL: $\underline{\text { http://www.jmir.org/2020/9/e16224/ }}$
doi: $\underline{10.2196 / 16224}$
PMID: $\underline{32975520}$

(C) Qiusha Min, Xin Wang, Bo Huang, Liangzhou Xu. Originally published in the Journal of Medical Internet Research (http://www.jmir.org), 25.09.2020. This is an open-access article distributed under the terms of the Creative Commons Attribution License (https://creativecommons.org/licenses/by/4.0/), which permits unrestricted use, distribution, and reproduction in any medium, provided the original work, first published in the Journal of Medical Internet Research, is properly cited. The complete 
bibliographic information, a link to the original publication on http://www.jmir.org/, as well as this copyright and license information must be included. 